International Journal of Pure and Applied Mathematics

Volume 105 No. 1 2015, 59-72

ISSN: 1311-8080 (printed version); ISSN: 1314-3395 (on-line version)

url: http://www.ijpam.eu

doi: http://dx.doi.org/10.12732/ijpam.v105i1.6

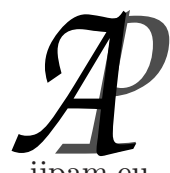

ijpam.eu

\title{
ALMOST CONTINUITY VIA TOPOLOGICAL GRILLS
}

\author{
N. Karthikeyan ${ }^{1}$, N. Rajesh ${ }^{2}$ \\ ${ }^{1}$ Department of Mathematics \\ Jeppiaar Engineering College \\ Chennai, 600119, TamilNadu, INDIA \\ ${ }^{2}$ Department of Mathematics \\ Rajah Serfoji Govt. College \\ Thanjavur, 613005, TamilNadu, INDIA
}

\begin{abstract}
The aim of this paper is to introduce and characterize a new class of functions called almost $\mathcal{G}$-precontinuous functions in ideal topological spaces by using $\mathcal{G}$-preopen sets.
\end{abstract}

AMS Subject Classification: 54D10

Key Words: ideal topological spaces, $\mathcal{G}$-preopen sets, almost $\mathcal{G}$-precontinuous functions

\section{Introduction}

The idea of grills on a topological space was first introduced by Choquet [8]. The concept of grills has shown to be a powerful supporting and useful tool like nets and filters, for getting a deeper insight into further studying some topological notions such as proximity spaces, closure spaces and the theory of compactifications and extension problems of different kinds (see [6],[7],[21] for details). In [17], Roy and Mukherjee defined and studied a typical topology associated rather naturally to the existing topology and a grill on a given topological space. Quite recently, Hatir and Jafari [10] have defined new classes of

Received: July 28, 2015

(C) 2015 Academic Publications, Ltd.

${ }^{\S}$ Correspondence author url: www.acadpubl.eu 
sets in a grill topological space and obtained a new decomposition of continuity in terms of grills. The aim of this paper is to introduce and characterize a new class of functions called almost $\mathcal{G}$-precontinuous functions in ideal topological spaces by using $\mathcal{G}$-preopen sets.

\section{Preliminaries}

Let $\mathrm{A}$ be a subset of a topological space $(X, \tau)$. We denote the closure of $A$ and the interior of $A$ by $\mathrm{Cl}(A)$ and $\operatorname{Int}(A)$, respectively. A subset $A$ of a topological space $(X, \tau)$ is said to be regular open [20] if $A=\operatorname{Int}(\mathrm{Cl}(A))$. A set $A \subset X$ is said to be $\delta$-open [22] if it is the union of regular open sets of $X$. The complement of a regular open (resp. $\delta$-open) set is called regular closed (resp. $\delta$-closed). The intersection of all $\delta$-closed sets of $(X, \tau)$ containing $\mathrm{A}$ is called the $\delta$-closure [22] of $\mathrm{A}$ and is denoted by $\mathrm{Cl}_{\delta}(A)$. A point $x \in X$ is called a $\theta$-cluster point of $\mathrm{A}$ if $\mathrm{Cl}(A) \cap A \neq \varnothing$ for every open set $V$ of $X$ containing $x$. The set of all $\theta$-cluster points of $\mathrm{A}$ is called the $\theta$-closure of $\mathrm{A}$ [22] and is denoted by $\mathrm{Cl}_{\theta}(A)$. If $A=\mathrm{Cl}_{\theta}(A)$, then $A$ is said to be $\theta$-closed [22]. The complement of $\theta$-closed set is said to be $\theta$-open [22]. A subset $A$ of a topological space $(X, \tau)$ is said to be preopen [4] (resp. semiopen [14], preopen [15], $\beta$-open [2]) if $A \subset \operatorname{Int}(\mathrm{Cl}(A)) \cup \mathrm{Cl}(\operatorname{Int}(A))(\operatorname{resp} . A \subset \mathrm{Cl}(\operatorname{Int}(A))$, $A \subset \operatorname{Int}(\mathrm{Cl}(A)), A \subset \mathrm{Cl}(\operatorname{Int}(\mathrm{Cl}(A))))$. The set of all regular open (resp. regular closed, $\delta$-open, $\delta$-closed, preopen, preopen) sets of $(X, \tau)$ is denoted by $R O(X)$ (resp. $R C(X), \delta O(X), \delta C(X), B O(X), P O(X))$. The definition of grill on a topological space, as given by Choquet [8], goes as follows: A non-null collection $\mathcal{G}$ of subsets of a topological space $(X, \tau)$ is said to be a grill on $X$ if

(1) $\theta \notin \mathcal{G}$,

(2) $A \in \mathcal{G}$ and $A \subset B$ implies that $B \in \mathcal{G}$,

(3) $A, B \subset X$ and $A \cup B \in \mathcal{G}$ implies that $A \in \mathcal{G}$ or $B \in \mathcal{G}$.

Definition 2.1. [17] Let $(X, \tau)$ be a topological space and $\mathcal{G}$ a grill on $X$. A mapping $\Phi: \mathcal{P}(X) \rightarrow \mathcal{P}(X)$ is defined as follows: $\Phi(A)=\Phi_{\mathcal{G}}(A, \tau)=\{x \in$ $X: A \cap U \in \mathcal{G}$ for every open set $\mathrm{U}$ containing $x\}$ for each $A \in \mathcal{P}(X)$. The mapping $\Phi$ is called the operator associated with the grill $\mathcal{G}$ and the topology $\tau$.

Definition 2.2. [17] Let $\mathcal{G}$ be a grill on a topological space $(X, \tau)$. Then we define a map $\Psi: \mathcal{P}(X) \rightarrow \mathcal{P}(X)$ by $\Psi(A)=A \cup \Phi(A)$ for all $A \in \mathcal{P}(X)$. The map $\Psi$ is a Kuratowski closure axiom. Corresponding to a grill $\mathcal{G}$ on 
a topological space $(X, \tau)$, there exists a unique topology $\tau_{\mathcal{G}}$ on $X$ given by $\tau_{\mathcal{G}}=\{U \subseteq X: \Psi(X \backslash U)=X \backslash U\}$, where for any $A \subset X, \Psi(A)=A \cup \Phi(A)=$ $\tau_{\mathcal{G}} \mathrm{Cl}(A)$. For any grill $\mathcal{G}$ on a topological space $(X, \tau), \tau \subset \tau_{\mathcal{G}}$. If $(X, \tau)$ is a topological space with a grill $\mathcal{G}$ on $X$, then we call it a grill topological space and denote it by $(X, \tau, \mathcal{G})$.

Definition 2.3. [10] A subset $S$ of a grill topological space $(X, \tau, \mathcal{G})$ is $\mathcal{G}$-preopen if $S \subset \operatorname{Int}(\Psi(S))$. The complement of a $\mathcal{G}$-preopen set is called a $\mathcal{G}$-preclosed set.

Definition 2.4. The intersection of all $\mathcal{G}$-preclosed sets containing $S \subset X$ is called the $\mathcal{G}$-preclosure of $S$ and is denoted by $p \mathrm{Cl}_{\mathcal{G}}(S)$. The family of all $\mathcal{G}$-preopen (resp. $\mathcal{G}$-preclosed) sets of $(X, \tau, \mathcal{G})$ is denoted by $\mathcal{G} P O(X)$ (resp. $\mathcal{G} P C(X))$. The family of all $\mathcal{G}$-preopen (resp. $\mathcal{G}$ - preclosed) sets of $(X, \tau, \mathcal{G})$ containing a point $x \in X$ is denoted by $\mathcal{G} P O(X, x)$ (resp. $\mathcal{G} P C(X, x)$ ).

Definition 2.5. A function $f:(X, \tau) \rightarrow(Y, \sigma)$ is said to be:

(1) precontinuous [4] if $f^{-1}(V)$ is preopen in $X$ for every open set $V$ of $Y$;

(2) almost continuous [19] if $f^{-1}(V)$ is open in $X$ for every regular open set $V$ of $X$;

(3) $R$-map [5] if $f^{-1}(V)$ is regular open in $X$ for every regular open set $V$ of $X$

(4) almost precontinuous [16] if $f^{-1}(V)$ is preopen in $X$ for every regular open set $V$ of $Y$.

Definition 2.6. A function $f:(X, \tau, \mathcal{G}) \rightarrow(Y, \sigma, \mathcal{G})$ is said to be $\mathcal{G}$ preirresolute if $f^{-1}(V)$ is $\mathcal{G}$-preopen in $X$ for every $\mathcal{G}$-preopen subset $V$ of $Y$.

Definition 2.7. $A$ function $f:(X, \tau, \mathcal{G}) \rightarrow(Y, \sigma)$ is said to be:

(1) $\mathcal{G}$-precontinuous [10] if $f^{-1}(V)$ is $\mathcal{G}$-preopen in $X$ for every open set $V$ of $Y$,

(2) weakly $\mathcal{G}$-precontinuous [12] if for each $x \in X$ if for each open subset $V$ in $Y$ containing $f(x)$, there exists $U \in \mathcal{G P O}(X, x)$ such that $f(U) \subset \mathrm{Cl}(V)$.

Definition 2.8. An ideal topological space $(X, \tau, \mathcal{G})$ is said to be:

(1) $\mathcal{G}$-pre- $T_{1}$ [13] (resp. $r-T_{1}$ [9]) if for each pair of distinct points $x$ and $y$ of $X$, there exist $\mathcal{G}$-preopen (resp. regular open) sets and $U$ and $V$ such that $x \in U, y \notin U$ and $x \notin V, y \in V$. 
(2) $\mathcal{G}-$ pre- $T_{2}$ [13] (resp. $r-T_{2}$ [9]) if for each pair of distinct points $x$ and $y$ of $X$, there exist $\mathcal{G}$-preopen (resp. regular open) sets $U$ and $V$ such that $x \in U, y \in V$ and $U \cap V=\varnothing$.

Lemma 2.9. The following statements are true:

(1) Let $A$ be a subset of a space $(X, \tau)$. Then $A \in P O(X)$ if and only if $s$ $\mathrm{Cl}(A)=\operatorname{Int}(\mathrm{Cl}(A))[11]$.

(2) $A$ subset $A$ of a space $(X, \tau)$ is $\beta$-open if and only if $\mathrm{Cl}(A)$ is regular closed [3].

\section{Almost $\mathcal{G}$-Precontinuous Functions}

Definition 3.1. A function $f:(X, \tau, \mathcal{G}) \rightarrow(Y, \sigma)$ is said to be:

(1) almost $\mathcal{G}$-precontinuous at a point $x \in X$ if for each open subset $V$ of $Y$ containing $f(x)$, there exists $U \in \mathcal{G} P O(X, x)$ such that $f(U) \subset \operatorname{Int}(\mathrm{Cl}(V))$;

(2) almost $\mathcal{G}$-precontinuous if it has this property at each point of $X$.

Remark 3.2. Almost $\mathcal{G}$-precontinuity implies weak $\mathcal{G}$-precontinuity and it is obvious that almost $\mathcal{G}$-precontinuity implied by $\mathcal{G}$-precontinuity. However, the converse of these implications is not true in general as the following examples show.

Example 3.3. Let $X=\{a, b, c\}, \tau=\{\varnothing,\{a\},\{b\},\{a, b\}, X\}, \sigma=$ $\{\varnothing,\{a\},\{a, b\}, X\}$ and $\mathcal{G}=\mathcal{P}(X) \backslash\{\varnothing,\{a\}\}$. Define a function $f:(X, \tau, \mathcal{G}) \rightarrow$ $(Y, \sigma)$ by $f(a)=b, f(b)=c$ and $f(c)=a$. Then $f$ is almost $\mathcal{G}$-precontinuous but not $\mathcal{G}$-precontinuous.

Example 3.4. Let $X=\{a, b, c\}, \tau=\{\varnothing,\{a\},\{b, c\}\}, \sigma=\{\varnothing,\{a\},\{b\}$, $\{a, b\}, X\}$ and $\mathcal{G}=\mathcal{P}(X) \backslash\{\varnothing,\{a\}\}$. Then the identity function $f:(X, \tau, \mathcal{G}) \rightarrow$ $(X, \sigma)$ is weakly $\mathcal{G}$-precontinuous but not almost $\mathcal{G}$-continuous.

Theorem 3.5. For a function $f:(X, \tau, \mathcal{G}) \rightarrow(Y, \sigma)$, the following statements are equivalent:

(1) $f$ is almost $\mathcal{G}$-precontinuous at $x \in X$;

(2) $x \in \operatorname{Int}\left(\Psi\left(f^{-1}(s \mathrm{Cl}(V))\right)\right)$ for every open set $V$ of $Y$ containing $f(x)$;

(3) $f^{-1}(V) \subset p \operatorname{Int}_{\mathcal{G}}\left(f^{-1}(s \mathrm{Cl}(V))\right)$ for every open set $V$ of $Y$;

(4) $p \mathrm{Cl}_{\mathcal{G}}\left(f^{-1}(s \operatorname{Int}(F))\right) \subset f^{-1}(F)$ for every closed set $F$ of $Y$ 
Proof. (1) $\Rightarrow(2)$ : Let $V$ be an open set of $Y$ containing $f(x)$. Then there exists $U \in \mathcal{G} P O(X, x)$ such that $f(U) \subset \operatorname{Int}(\mathrm{Cl}(V))=s \mathrm{Cl}(V)$. Then $U \subset f^{-1}(s \mathrm{Cl}(V))$. Since $U \in \mathcal{G} P O(X, x), x \in U \subset \operatorname{Int}\left(\Psi\left(f^{-1}(U)\right)\right) \subset$ $\left(\operatorname{Int}\left(\Psi\left(f^{-1}(s \mathrm{Cl}(V))\right)\right)\right.$

$(2) \Rightarrow(3)$ : Let $V$ be an open set of $Y$ containing $f(x)$ and $U$ an open set of $X$ containing $x$. Since $x \in \operatorname{Int}\left(\Psi\left(f^{-1}(s \mathrm{Cl}(V))\right)\right)$, we have $x \in f^{-1}(s \mathrm{Cl}(V)) \cap$ $\left(\operatorname{Int}\left(\Psi\left(f^{-1}(s \mathrm{Cl}(V))\right)\right)=p \operatorname{Int}_{\mathcal{G}}\left(f^{-1}(s \mathrm{Cl}(V))\right)\right.$. Hence

$$
f^{-1}(V) \subset p \operatorname{Int}_{\mathcal{G}}\left(f^{-1}(s \mathrm{Cl}(V))\right) .
$$

(3) $\Rightarrow(1)$ : Let $V$ be an open set of $Y$ containing $f(x)$, then $x \in f^{-1}(V) \subset$ $p \operatorname{Int}_{\mathcal{G}}\left(f^{-1}(s \mathrm{Cl}(V))\right)$. Set $U=p \operatorname{Int}_{\mathcal{G}}\left(f^{-1}(s \mathrm{Cl}(V))\right)$, then $U \in \mathcal{G} P O(X, x)$ such that $f(U) \subset s \mathrm{Cl}(V)$. This shows that $f$ is almost $\mathcal{G}$-precontinuous at $x$.

$(3) \Rightarrow(4)$ : Clear.

Theorem 3.6. For a function $f:(X, \tau, \mathcal{G}) \rightarrow(Y, \sigma)$ the following statements are equivalent:

(1) $f$ is almost $\mathcal{G}$-precontinuous;

(2) $f^{-1}(\operatorname{Int}(\mathrm{Cl}(V)) \in \mathcal{G P O}(X)$ for every open set $V$ of $Y$;

(3) $f^{-1}(\mathrm{Cl}(\operatorname{Int}(V)) \in \mathcal{G} P O(X)$ for every closed set $V$ of $Y$;

(4) $f^{-1}(V) \in \mathcal{G P O}(X)$ for every $V \in R O(Y)$;

(5) $f^{-1}(F) \in \mathcal{G P C}(X)$ for every $F \in R C(Y)$;

(6) for each $x \in X$ and each open set $V$ of $Y$ containing $f(x)$ there exists $U \in \mathcal{G P O}(X, x)$ such that $f(U) \subset s \mathrm{Cl}(V)$;

(7) $p \mathrm{Cl}_{\mathcal{G}}\left(f^{-1}(\mathrm{Cl}(\operatorname{Int}(F))) \subset f^{-1}(F)\right.$ for every closed set $F$ of $Y$;

(8) $p \mathrm{Cl}_{\mathcal{G}}\left(f^{-1}(A)\right) \subset f^{-1}(\mathrm{Cl}(A))$ for every $A \in B O(Y)$;

(9) $p \mathrm{Cl}_{\mathcal{G}}\left(f^{-1}(A)\right) \subset f^{-1}(\mathrm{Cl}(A))$ for every $A \in S O(Y)$;

$(10) f^{-1}(V) \subset p \operatorname{Int}_{\mathcal{G}}\left(f^{-1}(\operatorname{Int}(\mathrm{Cl}(V)))\right)$ for every open set $V \in P O(Y)$;

(11) $f\left(p \mathrm{Cl}_{\mathcal{G}}(A)\right) \subset \mathrm{Cl}_{\delta}(f(A))$ for every subset $A$ of $X$;

(12) $p \mathrm{Cl}_{\mathcal{G}}\left(f^{-1}(B)\right) \subset f^{-1}\left(\mathrm{Cl}_{\delta}(B)\right)$ for every subset $B$ of $Y$;

(13) $f^{-1}(F) \in \mathcal{G P C}(X)$ for every $F \in \delta C(Y)$;

(14) $f^{-1}(V) \in \mathcal{G} P O(X)$ for every $V \in \delta O(Y)$. 
Proof. (4) $\Rightarrow(5)$ : Let $F \in R C(Y)$. Then $Y \backslash F \in R O(Y)$. Take $x \in$ $f^{-1}(Y \backslash F)$, then $f(x) \in Y \backslash F$ and since $f$ is almost $b$ - $\mathcal{G}$-precontinuous, there exists $W_{x} \in \mathcal{G P O}(X, x)$ such that $x \in W_{x}$ and $f\left(W_{x}\right) \subset Y \backslash F$. Then $x \in$ $W_{x} \subset f^{-1}(Y \backslash F)$ so that $f^{-1}(Y \backslash F)=\underset{x \in f^{-1}(Y \backslash F)}{\cup} W_{x}$. Since any union of $\mathcal{G}$-preopen sets is $\mathcal{G}$-preopen $[10], f^{-1}(Y \backslash F)$ is $\mathcal{G}$-preopen in $X$ and hence $f^{-1}(F) \in \mathcal{G P C}(X)$.

$(5) \Rightarrow(11)$ : Let $A$ be a subset of $X$. Since $\mathrm{Cl}_{\delta}(f(A))$ if $\delta$-closed in $Y$, it is equal to $\cap\left\{F_{\alpha}: F_{\alpha}\right.$ is regular closed in $\left.Y, \alpha \in \Lambda\right\}$, where $\Lambda$ is an index set. From (5), we have $A \subset f^{-1}\left(\mathrm{Cl}_{\delta}(f(A))\right)=\cap\left\{f^{-1}\left(F_{\alpha}\right): \alpha \in \Lambda\right\} \in \mathcal{G} P C(X)$ and hence $p \mathrm{Cl}_{\mathcal{G}}(A) \subset f^{-1}\left(\mathrm{Cl}_{\delta}(f(A))\right)$. Therefore, we obtain $f\left(p \mathrm{Cl}_{\mathcal{G}}(A)\right) \subset \mathrm{Cl}_{\delta}(f(A))$.

$(11) \Rightarrow(12)$ : Set $A=f^{-1}(B)$ in $(11)$, then

$$
f\left(P \mathrm{Cl}_{\mathcal{G}}\left(f^{-1}(B)\right)\right) \subset \mathrm{Cl}_{\delta}\left(f\left(f^{-1}(B)\right)\right) \subset \mathrm{Cl}_{\delta}(B)
$$

and hence $p \mathrm{Cl}_{\mathcal{G}}\left(f^{-1}(B)\right) \subset f^{-1}\left(\mathrm{Cl}_{\delta}(B)\right)$.

$(12) \Rightarrow(13)$ : Let $F$ be $\delta$-closed set of $Y$, then $p \mathrm{Cl}_{\mathcal{G}}\left(f^{-1}(F)\right) \subset f^{-1}(F)$ so $f^{-1}(F) \in \mathcal{G} P C(X)$.

$(13) \Rightarrow(14)$ : Let $V$ be $\delta$-open set of $Y$, then $Y \backslash V$ is $\delta$-closed set in $Y$. This gives $f^{-1}(Y \backslash V) \in \mathcal{G P C}(X)$ and hence $f^{-1}(V) \in \mathcal{G P O}(X)$.

$(14) \Rightarrow(1)$ : Let $V$ be any regular open set of $Y$. Since $V$ is $\delta$-open in $Y$, $f^{-1}(V) \in \mathcal{G P O}(X)$ and hence from $f\left(f^{-1}(V)\right) \subset V=\operatorname{Int}(\mathrm{Cl}(V))$. Then $f$ is almost $\mathcal{G}$-precontinuous.

$(5) \Rightarrow(8)$ : Let $A$ be any $b$-open set in $Y$. Since $\mathrm{Cl}(A)$ is regular closed, $f^{-1}(\mathrm{Cl}(A))$ is $\delta$-closed and $f^{-1}(A) \subset f^{-1}(\mathrm{Cl}(A))$. Hence, $p \mathrm{Cl}_{\mathcal{G}}\left(f^{-1}(A)\right) \subset$ $f^{-1}(\mathrm{Cl}(A))$.

$(8) \Rightarrow(9)$ : obvious.

$(9) \Rightarrow(10)$ : Let $V$ be a preopen set. Then we have $V \subset \operatorname{Int}(\mathrm{Cl}(V))$ and $\mathrm{Cl}(\operatorname{Int}(Y \backslash V)) \subset Y \backslash V$. Moreover, since the set $\mathrm{Cl}(\operatorname{Int}(Y \backslash V))$ is semiopen, it follows that

$$
\begin{aligned}
X \backslash p \operatorname{Int}_{\mathcal{G}}\left(f^{-1}(\operatorname{Int}(\mathrm{Cl}(V)))\right)=p \mathrm{Cl}_{\mathcal{G}}\left(X \backslash f^{-1}(\operatorname{Int}(\mathrm{Cl}(V)))\right) \\
=p \mathrm{Cl}_{\mathcal{G}}\left(f^{-1}(Y \backslash \operatorname{Int}(\mathrm{Cl}(V)))\right)=p \mathrm{Cl}_{\mathcal{G}}\left(f^{-1}(\mathrm{Cl}(\operatorname{Int}(Y \backslash V)))\right) \\
\subset f^{-1}(\mathrm{Cl}(\operatorname{Int}(Y \backslash V))) \subset f^{-1}(Y \backslash V) \subset X \backslash f^{-1}(V) .
\end{aligned}
$$

Hence, we obtain $f^{-1}(V) \subset p \operatorname{Int}_{\mathcal{G}} \operatorname{Int}\left(f^{-1}(\operatorname{Int}(\mathrm{Cl}(V)))\right)$. 
$(10) \Rightarrow(4)$ : Let $V$ be a regular open set. Since $V$ is pre open, we get $f^{-1}(V) \subset p \operatorname{Int}_{\mathcal{G}}\left(f^{-1}(\operatorname{Int}(\mathrm{Cl}(V)))\right)=p \operatorname{Int}_{\mathcal{G}}\left(f^{-1}(V)\right)$. Hence $f^{-1}(V) \in \mathcal{G} P O(X)$.

The other implications are obvious.

Theorem 3.7. For a function $f:(X, \tau, \mathcal{G}) \rightarrow(Y, \sigma)$, the following statements are equivalent:

(1) $f$ is almost $\mathcal{G}$-precontinuous;

(2) $\left.\left.p \mathrm{Cl}_{\mathcal{G}}\left(f^{-1}(\mathrm{Cl}(\operatorname{Int}(\mathrm{Cl}(B))))\right)\right) \subset f^{-1}(\mathrm{Cl}(B))\right)$ for every open subset $B$ of $Y$;

(3) $\left.\left.p \mathrm{Cl}_{\mathcal{G}}\left(f^{-1}(\mathrm{Cl}(\operatorname{Int}(F)))\right)\right) \subset f^{-1}(F)\right)$ for every closed subset $F$ of $Y$;

(4) $\left.p \mathrm{Cl}_{\mathcal{G}}\left(f^{-1}(\mathrm{Cl}(V))\right)\right) \subset f^{-1}(\mathrm{Cl}(V))$ for every open subset $V$ of $Y$;

(5) $f^{-1}(V) \subset p \operatorname{Int}_{\mathcal{G}}\left(f^{-1}(s \mathrm{Cl}(V))\right)$ for every open subset $V$ of $Y$;

(6) $f^{-1}(V) \subset \operatorname{Int}\left(\Psi\left(f^{-1}(s \mathrm{Cl}(V))\right)\right)$ for every open subset $V$ of $Y$;

(7) $f^{-1}(V) \subset \operatorname{Int}\left(\Psi\left(f^{-1}(\operatorname{Int}(\mathrm{Cl}(V)))\right)\right)$ for every open subset $V$ of $Y$.

Proof. (1) $\Rightarrow(2)$ : Let $B$ be any subset of $Y$. Assume that $x \in X \backslash f^{-1}(\mathrm{Cl}(B))$. Then $f(x) \in Y \backslash \mathrm{Cl}(B)$ and there exists an open set $V$ containing $f(x)$ such that $V \cap B=\emptyset$; hence $\operatorname{Int}(\mathrm{Cl}(V)) \cap \mathrm{Cl}(\operatorname{Int}(\mathrm{Cl}(B)))=\emptyset$. Since $f$ is almost $\mathcal{G}$-precontinuous, there exists $U \in \mathcal{G} P O(X, x)$ such that $f(U) \subset \operatorname{Int}(\mathrm{Cl}(V))$.

Therefore, we have $U \cap f^{-1}(\mathrm{Cl}(\operatorname{Int}(\mathrm{Cl}(B))))=\emptyset$ and hence

$$
x \in X \backslash p \mathrm{Cl}_{\mathcal{G}}\left(f^{-1}(\mathrm{Cl}(\operatorname{Int}(\mathrm{Cl}(B)))) .\right.
$$

Thus, we obtain $\left.\left.p \mathrm{Cl}_{\mathcal{G}}\left(f^{-1}(\mathrm{Cl}(\operatorname{Int}(\mathrm{Cl}(B))))\right)\right) \subset f^{-1}(\mathrm{Cl}(B))\right)$.

$(2) \Rightarrow(3)$ : Let $F$ be any closed set of $Y$. Then we have

$$
\begin{aligned}
p \mathrm{Cl}_{\mathcal{G}}\left(f^{-1}(\mathrm{Cl}(\operatorname{Int}(\mathrm{Cl}(\operatorname{Int}(F)))))=p \mathrm{Cl}_{\mathcal{G}}\left(f^{-1}(\mathrm{Cl}(\operatorname{Int}(F)))\right)\right. \\
\\
\subset f^{-1}(\mathrm{Cl}(\operatorname{Int}(F))) \subset f^{-1}(F) .
\end{aligned}
$$

$(3) \Rightarrow(4)$ : For any open set $V$ of $Y, \mathrm{Cl}(V)$ is regular closed in $Y$ and we have $p \mathrm{Cl}_{\mathcal{G}}\left(f^{-1}(\mathrm{Cl}(V))\right)=p \mathrm{Cl}_{\mathcal{G}}\left(f^{-1}(\mathrm{Cl}(\operatorname{Int}(\mathrm{Cl}(V))))\right) \subset f^{-1}(\mathrm{Cl}(V))$.

$(4) \Rightarrow(5)$ : Let $V$ be any open set of $Y$. Then $Y \backslash \mathrm{Cl}(V)$ is open in $Y$ and we have $X \backslash p \operatorname{Int}_{\mathcal{G}}\left(f^{-1}(s \mathrm{Cl}(V))\right)=p \mathrm{Cl}_{\mathcal{G}}\left(f^{-1}(Y \backslash(s \mathrm{Cl}(V))) \subset f^{-1}(\mathrm{Cl}(Y \backslash \mathrm{Cl}(V))) \subset\right.$ $X \backslash f^{-1}(V)$. Therefore, we obtain $f^{-1}(V) \subset p \operatorname{Int}_{\mathcal{G}}\left(f^{-1}(s \mathrm{Cl}(V))\right)$. 
$(5) \Rightarrow(6)$ : Let $V$ be any open set of $Y$. Then we obtain $f^{-1}(V) \subset$ $p \operatorname{Int}_{\mathcal{G}}\left(f^{-1}(s \mathrm{Cl}(V))\right) \subset \operatorname{Int}\left(\Psi\left(f^{-1}(s \mathrm{Cl}(V))\right)\right)$.

$(6) \Rightarrow(1)$ : Let $x$ be any point of $X$ and $V$ any open set of $Y$ containing $f(x)$. Then $x \in f^{-1}(V) \subset \operatorname{Int}\left(\Psi\left(f^{-1}(s \mathrm{Cl}(V))\right)\right)$. It follows from Theorem 3.5 that $f$ is almost $\mathcal{G}$-precontinuous at any point $x$ of $X$. Therefore, $f$ is almost $\mathcal{G}$-precontinuous at any point $x$ of $X$.

$(6) \Rightarrow(7)$ : Clear.

Theorem 3.8. (1) A function $f:(X, \tau, \mathcal{P}(X) \backslash\{\varnothing\}) \rightarrow(Y, \sigma)$ is almost $\mathcal{G}$-precontinuous if and only if it is almost precontinuous. (2) A function $f:(X, \tau,\{X\}) \rightarrow(Y, \sigma)$ is almost $\mathcal{G}$-precontinuous if and only if it is almost continuous.

Proof. The proof follows from Corollaries 3.3 and 3.4 of [1].

Definition 3.9. A filterbase $\Lambda$ is said to be

$(1) \mathcal{G}$-preconvergent to a point $x$ in $X$ if for any $U \in \mathcal{G P O}(X, x)$, there exists $B \in \Lambda$ such that $B \subset U$.

(2) $r$-convergent to a point $x$ in $X$ if for any regular open set $U$ of $X$ containing $x$, there exists $B \in \Lambda$ such that $B \subset U$.

Theorem 3.10. If a function $f:(X, \tau, \mathcal{G}) \rightarrow(Y, \sigma)$ is almost $\mathcal{G}$ - precontinuous, then for each point $x \in X$ and each filter base $\Lambda$ in $X \mathcal{G}$-preconverging to $x$, the filter base $f(\Lambda)$ is $r$-convergent to $f(x)$.

Proof. Let $x \in X$ and $\Lambda$ be any filter base in $X \mathcal{G}$-preconverging to $x$. Since $f$ is $\mathcal{G}$-precontinuous, then for any open set $V$ of $(Y, \sigma)$ containing $f(x)$, there exists $U \in \mathcal{G} P O(X, x)$ such that $f(U) \subset V$. Since $\Lambda$ is $\mathcal{G}$ - preconverging to $x$, there exists $B \in \Lambda$ such that $B \subset U$. This means that $f(B) \subset V$ and hence the filter base $f(\Lambda)$ is convergent to $f(x)$.

Definition 3.11. $A$ sequence $\left(x_{n}\right)$ is said to be $\mathcal{G}$-preconvergent to a point $x$ if for every $\mathcal{G}$-preopen set $V$ containing $x$, there exists an index $n_{0}$ such that for $n \geqslant n_{0}, x_{n} \in V$.

Theorem 3.12. If a function $f:(X, \tau, \mathcal{G}) \rightarrow(Y, \sigma)$ is almost $\mathcal{G}$-precontinuous, then for each point $x \in X$ and each net $\left(x_{n}\right)$ which is $\mathcal{G}$-preconvergt to $x$, the net $\left(f\left(x_{n}\right)\right)$ is $r$-convergent to $f(x)$.

Proof. The proof is similar to that of Theorem 3.10. 
Theorem 3.13. If an injective function $f:(X, \tau, \mathcal{G}) \rightarrow(Y, \sigma)$ is almost $\mathcal{G}$-precontinuous and $(Y, \sigma)$ is $r$ - $T_{1}$, then $(X, \tau, \mathcal{G})$ is $\mathcal{G}$-pre $T_{1}$.

Proof. Suppose that $Y$ is $r-T_{1}$. For any distinct points $x$ and $y$ in $X$, there exist regular open sets $V$ and $W$ such that $f(x) \in V, f(y) \notin V, f(x) \notin W$ and $f(y) \in W$. Since $f$ is almost $\mathcal{G}$-precontinuous, $f^{-1}(V)$ and $f^{-1}(W)$ are $\mathcal{G}$-preopen subsets of $(X, \tau, \mathcal{G})$ such that $x \in f^{-1}(V), y \notin f^{-1}(V), x \notin f^{-1}(W)$ and $y \in f^{-1}(W)$. This shows that $(X, \tau, \mathcal{G})$ is $\mathcal{G}$-pre $T_{1}$.

Theorem 3.14. If $f:(X, \tau, \mathcal{G}) \rightarrow(Y, \sigma)$ is a almost $\mathcal{G}$-precontinuous injective function and $(Y, \sigma)$ is $r$ - $T_{2}$, then $(X, \tau)$ is $\mathcal{G}$-pre $T_{2}$.

Proof. For any pair of distinct points $x$ and $y$ in $X$, there exist disjoint regular open sets $U$ and $V$ in $Y$ such that $f(x) \in U$ and $f(y) \in V$. Since $f$ is almost $\mathcal{G}$-precontinuous, $f^{-1}(U)$ and $f^{-1}(V)$ are $\mathcal{G}$-preopen sets in $X$ containing $x$ and $y$, respectively. Therefore, $f^{-1}(U) \cap f^{-1}(V)=\varnothing$ because $U \cap V=\varnothing$. This shows that $(X, \tau, \mathcal{G})$ is $\mathcal{G}$-pre $T_{2}$.

Theorem 3.15. If $f:(X, \tau, \mathcal{G}) \rightarrow(Y, \sigma)$ is a almost continuous function and $g:(X, \tau, \mathcal{G}) \rightarrow(Y, \sigma)$ is a almost $\mathcal{G}$-precontinuous function and $Y$ is a $r$ - $T_{2}$-space, then the set $E=\{x \in X: f(x)=g(x)\}$ is $\mathcal{G}$-preclosed set in $(X, \tau, \mathcal{G})$.

Proof. If $x \in X \backslash E$, then it follows that $f(x) \notin g(x)$. Since $Y$ is $r$ - $T_{2}$, there exist disjoint regular open sets $V$ and $W$ of $Y$ such that $f(x) \in V$ and $g(x) \in W$. Since $f$ is almost continuous and $g$ is almost $\mathcal{G}$-precontinuous, then $f^{-1}(V)$ is open and $g^{-1}(W)$ is $\mathcal{G}$-preopen in $X$ with $x \in f^{-1}(V)$ and $x \in g^{-1}(W)$. Put $A=f^{-1}(V) \cap g^{-1}(W)$. By Corollary 3.1 of [1], $A$ is $\mathcal{G}$-preopen in $X$. Therefore, $f(A) \cap g(A)=\varnothing$ and it follows that $x \notin p \mathrm{Cl}_{\mathcal{G}}(E)$. This shows that $E$ is $\mathcal{G}$ preclosed in $X$.

Definition 3.16. A function $f:(X, \tau, \mathcal{G}) \rightarrow(Y, \sigma)$ is said to be faintly $\mathcal{G}$-precontinuous if for each $x \in X$ and each $\theta$-open set $V$ of $Y$ containing $f(x)$, there exists $U \in \mathcal{G} P O(X, x)$ such that $f(U) \subset V$.

Theorem 3.17. A function $f:(X, \tau, \mathcal{G}) \rightarrow(Y, \sigma)$ is faintly $\mathcal{G}$-precontinuous if and only if for every $\theta$-closed set $V$ of $Y f^{-1}(V) \in \mathcal{G} P C(X)$.

Theorem 3.18. The implications $(1) \Rightarrow(2) \Rightarrow(3) \Rightarrow(4) \Rightarrow(5)$ hold for the following properties of a function $f:(X, \tau, \mathcal{G}) \rightarrow(Y, \sigma)$ :

(1) $f$ is $\mathcal{G}$-precontinuous. 
(2) $f^{-1}\left(\mathrm{Cl}_{\delta}(B)\right)$ is $\mathcal{G}$-preclosed in $X$ for every subset $B$ of $Y$.

(3) $f$ is almost $\mathcal{G}$-precontinuous.

(4) $f$ is weakly $\mathcal{G}$-precontinuous.

(5) $f$ is faintly $\mathcal{G}$-precontinuous.

If, in addition, $Y$ is regular, then the five properties are equivalent of one another.

Proof. $(1) \Rightarrow(2)$ : Since $\mathrm{Cl}_{\delta}(B)$ is closed in $Y$ for every subset $B$ of $Y$, by Theorem 3.6, $f^{-1}\left(\mathrm{Cl}_{\delta}(B)\right)$ is $\mathcal{G}$-preclosed in $X$.

$(2) \Rightarrow(3)$ : For any subset $B$ of $Y, f^{-1}\left(\mathrm{Cl}_{\delta}(B)\right)$ is $\mathcal{G}$-preclosed in $X$ and hence we have $p \mathrm{Cl}_{\mathcal{G}}\left(f^{-1}(B)\right) \subset p \mathrm{Cl}_{\mathcal{G}}\left(f^{-1}\left(\mathrm{Cl}_{\delta}(B)\right)=f^{-1}\left(\mathrm{Cl}_{\delta}(B)\right)\right.$. It follows from Theorem 3.6 that $f$ is almost $\mathcal{G}$-precontinuous.

$(3) \Rightarrow(4)$ : This is obvious.

$(4) \Rightarrow(5)$ : Let $F$ be any $\theta$-closed set of $Y$. It follows from 3.17 that $p \mathrm{Cl}_{\mathcal{G}}\left(f^{-1}(F)\right) \subset f^{-1}\left(\mathrm{Cl}_{\theta}(F)\right)=f^{-1}(F)$. Therefore, $f^{-1}(F)$ is $\mathcal{G}$-preclosed in $X$ and hence $f$ is faintly $\mathcal{G}$-precontinuous.

Suppose that $Y$ is regular. We prove that $(5) \Rightarrow(1)$. Let $V$ be any open set of $Y$. Since $Y$ is regular, $V$ is $\theta$-open in $Y$. By the faint pre continuity of $f, f^{-1}$ is $\mathcal{G}$-preopen in $X$. Therefore, $f$ is $\mathcal{G}$-precontinuous.

Definition 3.19. A function $f:(X, \tau, \mathcal{G}) \rightarrow(Y, \sigma)$ is said to be $\mathcal{G}$-preopen if $f(U) \in P O(Y)$ for every $\mathcal{G}$-preopen set $U$ of $X$.

Theorem 3.20. If a function $f:(X, \tau, \mathcal{G}) \rightarrow(Y, \sigma)$ is $\mathcal{G}$-preopen and weakly $\mathcal{G}$-precontinuous, then $f$ is almost $\mathcal{G}$-precontinuous.

Proof. Let $x \in X$ and let $V$ be an open set of $Y$ containing $f(x)$. Since $f$ is weakly $\mathcal{G}$-precontinuous, there exists $U \in \mathcal{G} P O(X, x)$ such that $f(U) \subset \mathrm{Cl}(V)$. Since $f$ is $\mathcal{G}$-preopen, $f(U) \subset \operatorname{Int}(\mathrm{Cl}(f(U))) \subset \operatorname{Int}(\mathrm{Cl}(V))$; hence $f$ is almost $\mathcal{G}$-precontinuous.

Theorem 3.21. Let $f:(X, \tau, \mathcal{G}) \rightarrow(Y, \sigma)$ be a function and $g: X \rightarrow$ $X \times Y$ the graph function defined by $g(x)=(x, f(x))$ for every $x \in X$. Then $g$ is almost $\mathcal{G}$-precontinuous if and only if $f$ is almost $\mathcal{G}$-precontinuous. 
Proof. Let $x$ be any point of $X$ and $V$ any regular open set of $Y$ containing $f(x)$. Then we have $g(x)=(x, f(x)) \in X \times V$ is regular open in $X \times Y$. Since $g$ is almost $\mathcal{G}$-precontinuous, there exists $U \in \mathcal{G} P O(X, x)$ such that $g(U) \subset X \times V$. Therefore, we obtain $f(U) \subset V$; hence $f$ is almost $\mathcal{G}$-precontinuous. Conversely, let $x \in X$ and $W$ be a regular open set of $X \times Y$ containing $g(x)$. There exist a regular open set $U_{1}$ in $X$ and a regular open set $V$ in $Y$ such that $U_{1} \times V \subset W$. Since $f$ is almost $\mathcal{G}$-precontinuous, there exist $U_{2} \in \mathcal{G} P O(X, x)$ such that $f\left(U_{2}\right) \subset V$. Put $U=U_{1} \cap U_{2}$, then we obtain $x \in U \in \mathcal{G} P O(X)$ and $g(U) \subset U \times V \subset W$. This shows that $g$ is almost $\mathcal{G}$-precontinuous.

Theorem 3.22. Let $f:(X, \tau, \mathcal{G}) \rightarrow(Y, \sigma, \mathcal{G})$ and $g:(Y, \sigma, \mathcal{G}) \rightarrow(Z, \eta)$ be functions. Then the composition $g \circ f:(X, \tau, \mathcal{G}) \rightarrow(Z, \eta)$ is almost $\mathcal{G}$ precontinuous if $f$ and $g$ satisfy one of the following conditions:

(1) $f$ is almost $\mathcal{G}$-precontinuous and $g$ is $R$-map.

(2) $f$ is $\mathcal{G}$-preirresolute and $g$ is almost $\mathcal{G}$-precontinuous.

(3) $f$ is $\mathcal{G}$-precontinuous and $g$ is almost continuous

Proof. Clear.

Definition 3.23. A topological space $(X, \tau)$ is said to be:

(1) almost regular [18] if for any regular closed set $F$ of $X$ and any point $x \in X \backslash F$ there exist disjoint open sets $U$ and $V$ such that $x \in U$ and $F \subset V$.

(2) semi-regular if for any open set $U$ of $X$ and each point $x \in U$ there exists a regular open set $V$ of $X$ such that $x \in V \subset U$.

Theorem 3.24. If $f:(X, \tau, \mathcal{G}) \rightarrow(Y, \sigma)$ is a weakly $\mathcal{G}$-precontinuous function and $Y$ is almost regular, then $f$ is almost $\mathcal{G}$-precontinuous.

Proof. Let $x \in X$ and let $V$ be any open set of $Y$ containing $f(x)$. By the almost regularity of $Y$, there exists a regular open set $G$ of $Y$ such that $f(x) \in G \subset$ $\mathrm{Cl}(G) \subset \operatorname{Int}(\mathrm{Cl}(V))[[18]$, Theorem 2.2]. Since $f$ is weakly $\mathcal{G}$-precontinuous, there exists $U \in \mathcal{G P O}(X, x)$ such that $f(U) \subset \mathrm{Cl}(G) \subset \operatorname{Int}(\mathrm{Cl}(V))$. Therefore, $f$ is almost $\mathcal{G}$-precontinuous.

Theorem 3.25. If $f:(X, \tau, \mathcal{G}) \rightarrow(Y, \sigma)$ is an almost $\mathcal{G}$-precontinuous function and $Y$ is semi-regular, then $f$ is $\mathcal{G}$-precontinuous. 
Proof. Let $x \in X$ and let $V$ be any open set of $Y$ containing $f(x)$. By the semi-regularity of $Y$, there exists a regular open set $G$ of $Y$ such that $f(x) \in G \subset V$. Since $f$ is almost $\mathcal{G}$-precontinuous, there exists $U \in \mathcal{G} P O(X, x)$ such that $f(U) \in \operatorname{Int}(\operatorname{Cl}(G))=G \subset V$ and hence $f$ is $\mathcal{G}$-precontinuous.

Definition 3.26. A $\mathcal{G}$-prefrontier of a subset $A$ of $(X, \tau, \mathcal{G})$, denoted by $b \mathcal{G} \operatorname{Fr}(A)$, is defined by $p \mathrm{Cl}_{\mathcal{G}}(A) \cap p \mathrm{Cl}_{\mathcal{G}}(X \backslash A)$.

Theorem 3.27. The set of all points $x \in X$ in which a function $f$ : $(X, \tau, \mathcal{G}) \rightarrow(Y, \sigma)$ is not almost $\mathcal{G}$-precontinuous is identical with the union of $\mathcal{G}$-prefrontier of the inverse images of regular open sets containing $f(x)$.

Proof. Suppose that $f$ is not almost $\mathcal{G}$-precontinuous at $x \in X$. Then there exists a regular open set $V$ of $Y$ containing $f(x)$ such that $U \cap\left(X \backslash f^{-1}(V)\right) \neq$ $\emptyset$ for every $U \in \mathcal{G P O}(X, x)$. Therefore, we have $x \in p \mathrm{Cl}_{\mathcal{G}}\left(X \backslash f^{-1}(V)\right)=$ $X \backslash p \operatorname{Int}_{\mathcal{G}}\left(f^{-1}(V)\right)$ and $x \in f^{-1}(V)$. Thus, we obtain $x \in p_{\mathcal{G}} F r\left(f^{-1}(U)\right)$. Conversely, suppose that $f$ is almost $\mathcal{G}$-precontinuous at $x \in X$ and let $V$ be a regular open set of $Y$ containing $f(x)$. Then there exists $U \in \mathcal{G} P O(X, x)$ such that $U \subset f^{-1}(V)$. That is $x \in p \operatorname{Int}_{\mathcal{G}}\left(f^{-1}(V)\right)$. Therefore, $x \in X \backslash$ $p \mathcal{G} F r\left(f^{-1}(V)\right)$.

Theorem 3.28. If $g:(X, \tau, \mathcal{G}) \rightarrow(Y, \sigma)$ is almost $\mathcal{G}$-precontinuous and $S$ is $\delta$-closed set of $X \times Y$, then $p_{X}(S \cap \mathcal{G}(g))$ is $\mathcal{G}$-preclosed in $X$, where $p_{X}$ represents the projection of $X \times Y$ onto $X$ and $\mathcal{G}(g)$ denotes the graph of $g$.

Proof. Let $S$ be any $\delta$-closed set of $X \times Y$ and $x \in p \mathrm{Cl}_{\mathcal{G}}\left(p_{X}(S \cap \mathcal{G}(g))\right.$. Let $U$ be any open set of $X$ containing $x$ and $V$ any open set of $Y$ containing $g(x)$. Since $g$ is almost $\mathcal{G}$-precontinuous, we have $x \in g^{-1}(V) \subset$ $p \operatorname{Int}_{\mathcal{G}}\left(g^{-1}(\operatorname{Int}(\mathrm{Cl}(V)))\right)$ and $\left.U \cap p \operatorname{Int}_{\mathcal{G}}\left(g^{-1}(\operatorname{Int}(\mathrm{Cl}(V)))\right)\right) \in \mathcal{G} P O(X, x)$. Since $x \in p \mathrm{Cl}_{\mathcal{G}}\left(p_{x}(S \cap G(g)),\left(U \cap p \operatorname{Int}_{\mathcal{G}}\left(g^{-1}(\operatorname{Int}(\mathrm{Cl}(V)))\right)\right) \cap \cap p_{X}(S \cap G(g))\right.$ contains some point $u$ of $X$. This implies that $(u, g(u)) \in S$ and $g(u) \in \operatorname{Int}(\mathrm{Cl}(V))$. Thus, we have $\emptyset \neq(U \times \operatorname{Int}(\mathrm{Cl}(V)) \cap S \subset \operatorname{Int}(\mathrm{Cl}(U \times V)) \cap S$ and hence $(x, g(x)) \in \mathrm{Cl}_{\delta}(S)$. Since $S$ is $\delta$-closed, $(x, g(x)) \in p_{X}(S \cap G(g))$ and $x \in$ $p_{X}(S \cap G(g))$. Then $p_{X}(S \cap G(g))$ is $\mathcal{G}$-preclosed.

Corollary 3.29. If $f:(X, \tau, \mathcal{G}) \rightarrow(Y, \sigma)$ has a $\delta$-closed graph and $g$ : $(X, \tau, \mathcal{G}) \rightarrow(Y, \sigma)$ is almost $\mathcal{G}$-precontinuous, then the set $\{x \in X: f(x)=$ $g(x)\}$ is $\mathcal{G}$-preclosed in $X$.

Proof. Since $G(f)$ is $\delta$-closed and $p_{X}(G(f) \cap G(g))=\{x \in X: f(x)=g(x)\}$ it follows from Theorem 3.28 that $\{x \in X: f(x)=g(x)\}$ is $\mathcal{G}$-preclosed in $X$. 
Theorem 3.30. If for each pair of distinct $x_{1}$ and $x_{2}$ in an ideal topological space $(X, \tau, \mathcal{G})$ there exists a function $f$ of $X$ into a Hausdorff space $Y$ such that $f\left(x_{1}\right) \neq f\left(x_{2}\right), f$ is weakly $\mathcal{G}$-precontinuous and $f$ is almost $\mathcal{G}$-precontinuous at $x_{2}$, then $X$ is $\mathcal{G}$-pre- $T_{2}$.

Proof. Since $Y$ is Hausdorff, if for each pair of distinct point $x_{1}$ and $x_{2}$ there exist disjoint open sets $V_{1}$ and $V_{2}$ of $Y$ containing $f\left(x_{1}\right)$ and $f\left(x_{2}\right)$, respectively; hence $\mathrm{Cl}\left(V_{1}\right) \cap \operatorname{Int}\left(\mathrm{Cl}\left(V_{2}\right)\right)=\emptyset$. Since $f$ is weakly $\mathcal{G}$-precontinuous at $x_{1}$, there exists $U_{1} \in \mathcal{G P O}\left(X, x_{1}\right)$ such that $f\left(U_{1}\right) \subset \mathrm{Cl}\left(V_{1}\right)$. Since $f$ is almost $\mathcal{G}$-precontinuous at $x_{2}$, there exists $U_{2} \in \mathcal{G P O}\left(X, x_{2}\right)$ such that $f\left(U_{2}\right) \subset \operatorname{Int}\left(\mathrm{Cl}\left(V_{2}\right)\right)$. Therefore, we obtain $U_{1} \cap U_{2}=\emptyset$. This shows that $X$ is $\mathcal{G}$-pre- $T_{2}$.

\section{References}

[1] A. Al-Omari and T. Noiri, Decomposition of continuity via grills, Jordan J. Math and Stat., 4, No. 1 (2011), 33-46.

[2] M. E. Abd El-Monsef, S. N. El-Deep and R. A. Mahmoud, beta-open sets and beta-continuous functions Bull. Fac. Sci. Assiut Univ. A, 12 (1983), 77-90.

[3] D. Andrijevic, Semi-preopen sets, Math. Vesnik, 38 (1986), 24-32.

[4] D. Andrijevic, On preopen sets, Math. Vesnik, 48 (1996), 59-64.

[5] D.Carnahan, Some properties related to compactness in topological spaces, Ph.D.Thesis, Univ. Arkansas (1973).

[6] K. C. Chattopadhyay, O. Njastad and W. J. Thron, Merotopic spaces and extensions of closure spaces, Can. J. Math., 35, No. 4 (1983),613-629.

[7] K. C. Chattopadhyay and W. J. Thron, Extensions of closure spaces, Can. J. Math., 29, No. 6 (1977),1277-1286.

[8] G. Choqet, Sur les notions de filter et grill, Comptes Rendus Acad. Sci. Paris, 224 (1947), 171-173.

[9] E. Ekici, Generalization of perfectly continuous, Regular set-connected and clopen functions, Acta. Math. Hungar., 107, No. 3 (2005), 193-206.

[10] E. Hatir and S. Jafari, On some new calsses of sets and a new decomposition of continuity via grills, J. Adv. Math. Studies, 3, No. 1 (2010), 33-40. 
[11] D. S. Jankovic, A note on mappings of extremally disconnected spaces, Acta Math. Hungar., 46 (1985), 8392.

[12] N. Karthikeyan and N. Rajesh, On Weakly G-precontinuous functions (sub- mitted).

[13] N. Karthikeyan and N. Rajesh, Some New separation axioms in ideal topolog- ical space (submitted).

[14] N. Levine, Semi-open sets and semi-continuity in topological spaces, Amer. Math. Monthly, 70 (1963), 36-41.

[15] A. S. Mashhour, M. E. Abd El-Monsef and S. N. El-Deep, On precontinuous and weak precontinuous mappings, Proc. Math. Phys. Soc. Egypt, 53 (1982), 47-53.

[16] A. Nasif, T. Noiri, Some weak forms of almost continuity, Acta Math. Hungar., 74 (1997), 211-219.

[17] B. Roy and M. N. Mukherjee, On a typical topology induced by a grill, Soochow J. Math., 33 (4) (2007), 771-786.

[18] M. K. Singal and S. P. Arya, On almost regular spaces, Glasnik Mat., 4, No. 24 (1969), 89-99.

[19] M. K. Singal and A. R. Singal, Almost-continuous mappings, Yokohama Math. J., 16 (1968), 63-73.

[20] M. Stone, Applications of the theory of boolean rings to general topology, Trans. Amer. Math. Soc., 41 (1937), 374-381.

[21] W. J. Thron, Proximity structure and grills, Math. Ann., 206 (1973), 3562.

[22] N. V. Velicko, H-closed topological spaces, Amer. Math. Soc. Transl.78, No. 2 (1968), 103-118. 